\title{
A NOTE ON ONE-PARAMETER GROUPS OF AUTOMORPHISMS
}

\author{
A.B. THAHEEM \\ AND \\ NOOR MOHAMMAD \\ Department of Mathematics \\ Quiad-i-Azam University \\ Isl amabad, Pakistan
}

(Received July 7, 1988 and in revised form September 1, 1988)

ABSTRACT. Let $\left\{\alpha_{t}: t \in R\right\}$ and $\left\{\beta_{t}: t \in R\right\}$ be two commuting one-parameter groups of *-automorphisms of a von Neumann algebra $M$ such that $\alpha_{t}+\alpha_{-t}=\beta_{t}+\beta_{-t}$ for all

$t \in R$. The purpose of this note is to provide a simple and short proof of the central decomposition result: $\alpha_{t}=\beta_{t}$ on $M p$ and $\alpha_{t}=\beta_{-t}$ on $M(1-p)$ for a central projection $p \in M$, without using the theory of spectral subspaces.

KEY WORDS AND PHRASES. Automorphism groups, central projections, invariant projections.

1980 AMS SUBJECT CLASSIFICATION CODES. Primary 46L10, Secondary 47C15

\section{INTRODUCTION}

In [1], A. Van Daele, L. Vanheeswijck and one of the authors proved that if $\left\{\alpha_{t}: t \in R\right\}$ and $\left\{\beta_{t}: t \in R\right\}$ are strongly continuous one-parameter groups of *-automorphisms of a von Neumann algebra M satisfying the equation: $\alpha_{t}+\alpha_{-t}=$ $B_{t}+\beta_{-t}$ for all $t \in R$, then $M$ can be decomposed into subalgebras $M p$ and $M(1-p)$ for a central projection $p$ in $M$ such that $\alpha_{t}=\beta_{t}$ on $M p$ and $\alpha_{t}=\beta_{-t}$ on $M(1-p), t \in R$. The proof of this result is very technical and fairly long. It depends on Arveson's theory of spectral subspaces ([2], [3]) and as such it lacks a proper emphasis on the decomposition itself. However, there are important situations where it is enough to consider the commuting automorphism groups (see, for instance [4], [5]). The purpose of this note is to provide a simple proof of this decomposition result for commuting automorphism groups without using the theory of spectral subspaces. We use simple algebraic techniques to obtain the proof. of course, in doing so we lose the generality of the result but on the other hand we get a simple proof. For more details concerning the origin of this operator equation, its applications and related decomposition results, we refer to [6], [7], [8], [9], [10], [4] and [5]. 


\section{MAIN RESULTS}

The following is an important decomposition result for commuting automorphisms ( [10], [1 1]).

PROPOSITION 2.1. Let $\alpha, \beta$ be commuting *-automorphisms of a von Neumann algebra $M$ such that $\alpha+\alpha^{-1}=\beta+\beta^{-1}$. Then there exists a central projection $p$ in $M$ such that $\alpha=\beta$ on $M p$ and $\alpha=\beta^{-1}$ on $M(1-p)$.

In this paper, however, we use another version (Proposition 2.2) of the above result for the sake of clarity. The essential idea is that when we consider the *-automorphism $\alpha \beta$ on $M$, then by [12], $N(\alpha \beta-1)+R(\alpha \beta-1)$ is $\sigma$ - weakly dense in $M$ where $N(\alpha \beta-1)$ and $R(\alpha \beta-1)$ denote, respectively, the null space and the range space of the operators under consideration. $R(\alpha \beta-1) \subseteq N(\alpha-\beta)$ and the subalgebra $L$ (say) generated by $R(\alpha \beta-1)$ is a two-sided ideal in $M$ and there exists a largest central projection $P_{0}$ in $M$ such that $L=M p_{0}, M p_{0} \subseteq N(\alpha-\beta)$ and $p_{0}$ is $\alpha$, $\beta$ - invariant (that is $\left.\alpha\left(p_{0}\right)=\beta\left(p_{0}\right)=p_{0}\right)$. In other words, $\alpha=\beta$ on $\mathrm{Mp}_{0}$ (see for instance [10], [11]). Simllarly, by considering the orthogonal ideal $\mathrm{L}^{\perp}$ (note that $L^{\perp} \subseteq \mathrm{N}(\alpha \beta-1)$ ), we get a largest $\alpha, \beta$ - invariant central projection $q_{0}$ in $M$ such that $\alpha=B^{-1}$ on $\mathrm{Mq}_{0}$ and the orthogonality relation implies that

$\left(1-p_{0}\right)\left(1-q_{0}\right)=0$. For more detalls, we may refer to [10] and [11]. Thus we may have the following alternative version of Proposition 2.1.

Proposition 2.2. Let $\alpha, \beta$ be commuting *-automorphisms of a von Neumann algebra $M$ such that $\alpha+\alpha^{-1}=\beta+\beta^{-1}$. Among the projections $p \in M$ (respectively $q \in M$ ) such that $\alpha=\beta$ on $M p$ (respectively $\alpha=\beta^{-1}$ on $M q$ ) there exists a largest central projection $p_{0}$ in $M$ (respectively $q_{0}$ in $M$ ) such that $p_{0}$ and $q_{0}$ are $\alpha$, $\beta-$ invariant and $\left(1-p_{0}\right)\left(1-q_{0}\right)=0$.

We now come to our main result.

THEOREM 2.3. Let $\left\{\alpha_{t}: t \in R\right\}$ and $\left\{\beta_{t}: t \in R\right\}$ be two commuting one-parameter groups of *-automorphisms of a von Neumann algebra $M$ such that $\alpha_{t}+\alpha_{-t}=\beta_{t}+\beta_{-t}$ for all $t \in R$. Then there exists a central projection $p$ in $M$ such that

$$
\begin{aligned}
& \alpha_{t}(p)=\beta_{t}(p)=\text { for all } t \in R \text { and } \alpha_{t}=\beta_{t} \text { on } M p \text { and } \alpha_{t}=\beta_{-t} \text { on } M(1-p) \text {. } \\
& \text { PROOF. By Proposition 2.2, let } p_{n}, n \in N \text {, be the largest } \alpha_{2}, \beta_{2}-n
\end{aligned}
$$
-invariant central projection in $M$ such that

$$
{ }_{2}-\mathrm{n}={ }_{2}-\mathrm{n} \text { on } \mathrm{Mp}_{\mathrm{n}}
$$


Similarly, we let $q_{n}, n \in N$, be the largest $\alpha_{2}-n, \beta_{2}-n$-invariant central projection in $M$ such that

$$
\alpha_{2}-\mathrm{n}=\mathrm{B}_{2}-\mathrm{n} \text { on } \mathrm{Mq}_{\mathrm{n}}
$$

Then $\left(1-p_{n}\right)\left(1-q_{n}\right)=0$ for any $n \in N$. The sequence $\left\{p_{n}\right\}$ is decreasing because $\alpha_{2}-(n+1)=\beta_{2}-(n+1)$ on $M_{n+1}$ implies that their squares $\alpha_{2}-n$ and $\beta_{2}-n$ coincide on $M p_{n+1}$ and by maximality $p_{n+1}<p_{n}$. Similarly the sequence $\left\{q_{n}\right\}$ is decreasing.

Put $\mathrm{p}=\lim _{\mathrm{n} \rightarrow \infty} \mathrm{p}_{\mathrm{n}}$ and $\mathrm{q}=\lim _{\mathrm{n} \rightarrow \infty} \mathrm{q}_{\mathrm{n}}$. Then clearly $(1-\mathrm{p})(1-\mathrm{y})=0$. Since $\mathrm{p}_{\mathrm{n}}$ is $\alpha_{2}-\mathrm{m}, B_{2}^{-m}$-invariant for $n \geqslant m$, it follows that $p$ is $x_{2} m$, ${ }^{2} 2_{-}-$-invariant for any $m \in N$. The density of the set $\left\{k 2^{-m}: k \in Z, m \in N\right\}$ in $R$ and the continuity imply that $p$ is $\alpha_{t}$, $\beta_{t}$-invariant for any $t \in R$. Similarly $q$ is $\alpha_{t}$, $\beta_{t}$-invariant. Also $\alpha_{k 2^{-}}(x)=k_{k 2^{-}}(x)$ for $x \in M p \subseteq M_{m}$. Again by the density of the set

$\left\{k 2^{-m}: k \in Z, m \in N\right\}$, we conclude that $\alpha_{t}=\beta_{t}$ on Mp for any $t \in R$.

Similarly, $\alpha_{t}=\beta_{-t}$ on Mq. As $(1-q)(1-p)=0$ then $M(1-p) \subseteq M q$ and this completes the proof of the theorem.

ACKNOWLEDGEMENTS. The authors would like to thank Professor Abdus Salam, the International Atomic Energy Agency and UNESCO for hospitality at the International Centre for Theoretical Physics, Trieste.

\section{REFERENCES}

1. ThaHEEM, A.B., DAELE, A. VAN. and VANHEESWIJCK, L., A Result on Two Oneparameter Groups of Automorphisms, Math Scand. 51 (1982), 261-274.

2. ARVESON, W., On Groups of Automorphisms of Operator Algebras, J. Funct. Analy. $15(1974), 217-243$.

3. DAELE, A. VAN., Arveson's Theory of Spectral Subspaces, Nieuw Arch. Wisk. 27 (1979), 215-237.

4. HAAGERUP, U. and SKAU, F., Geometric Aspects of the Tomita-Takesaki Theory II, Math Scand. 48 (1981), 241-252.

5. HAAGERUP, U. and OLSEN, H., Tomita-Takesaki Theory for Jordan Algebras, J. Operator Theory 11 (1984), 343-364

6. DAELE, A. VAN., A New Approach to the Tomita-Takesaki Theory of Generalized Hilbert Algebras, J. Funct. Analy. 15 (1974), 373-393,

7. THAHEEM, A.B., A Bounded Map Assiciated $t$, a One-parameter Group of *Automorphisms of a von Neumann Algebra, Glasyow Math. J. 25 (1984), 135-140.

8. THAHEEM, A.B., On the Operator Fquation $\alpha+\alpha^{-1}=\beta+\beta^{-1}$, Internat. J. Math. \& Math. Sci. 9 (1986), 767-770. 
9. CIORǍNESCU, I. and ZSIDó, I., Analytic Generators for One-parameter Groups, Tôhoku Math. J. (2) 28 (1976) 327-362.

10. THAHEEM, A. B., Decomposition of a von Neumann Algebra, Rend. Sem. Mat. Univ. Padova 65(1981), 1-7.

11. AWAMI, M. and THAHEEM, A. B., A Short Proof of a Decomposition Theorem of a von Neumann Algebra, Proc. Amer. Math. Soc. 92 (1984), 81-82.

12. THAHEEM, A. B., Decomposition of a von Neumann Algebra Relative to a *Aut omorphism, Proc. Edinburgh Math. Soc. (2) 22 (1979), 9-10. 


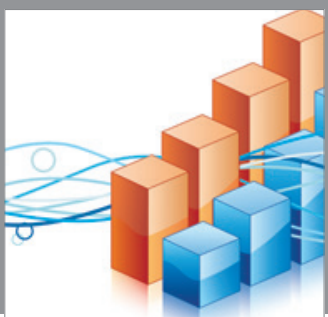

Advances in

Operations Research

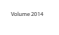

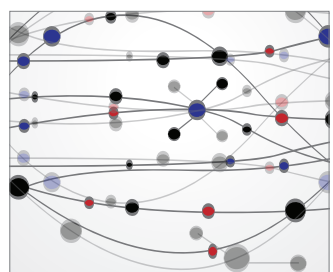

\section{The Scientific} World Journal
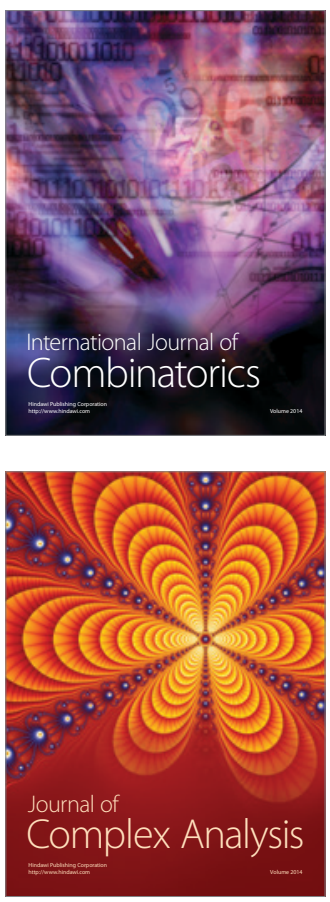

International Journal of

Mathematics and

Mathematical

Sciences
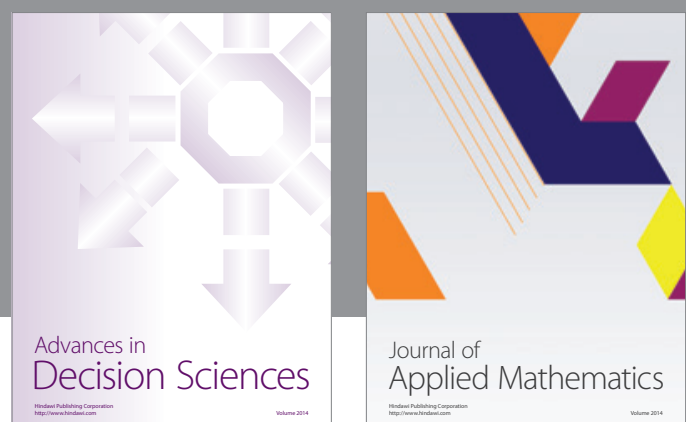

Journal of

Applied Mathematics
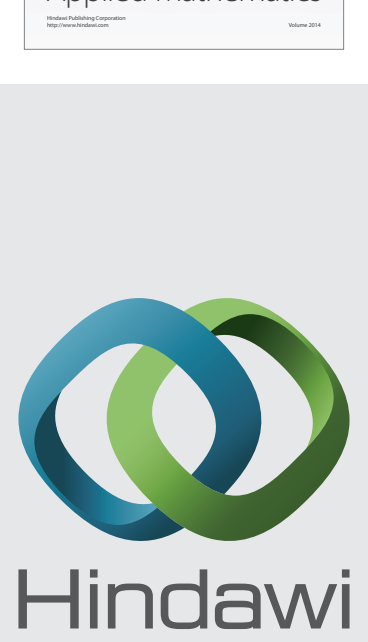

Submit your manuscripts at http://www.hindawi.com
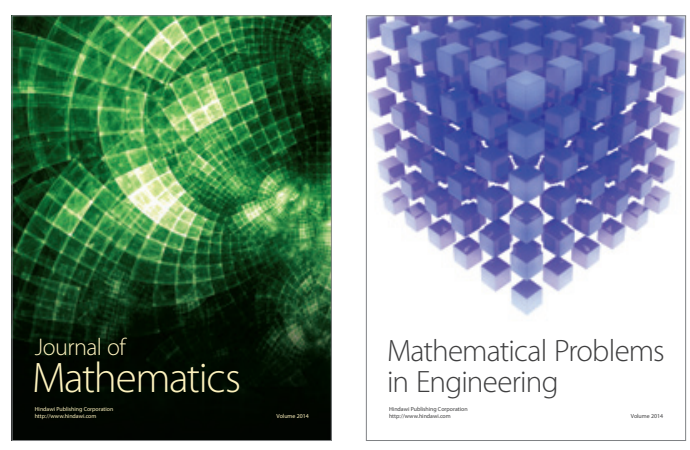

Mathematical Problems in Engineering
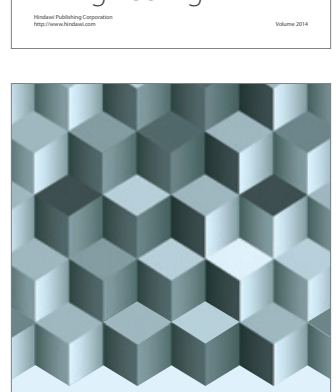

Journal of

Function Spaces
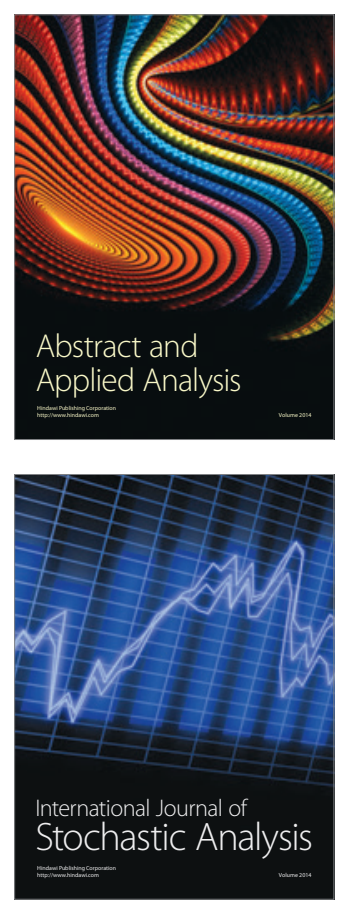

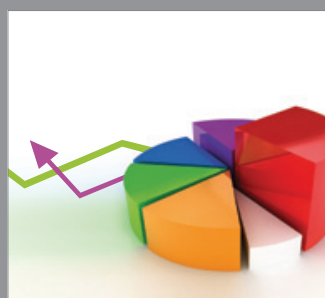

ournal of

Probability and Statistics

Promensencen
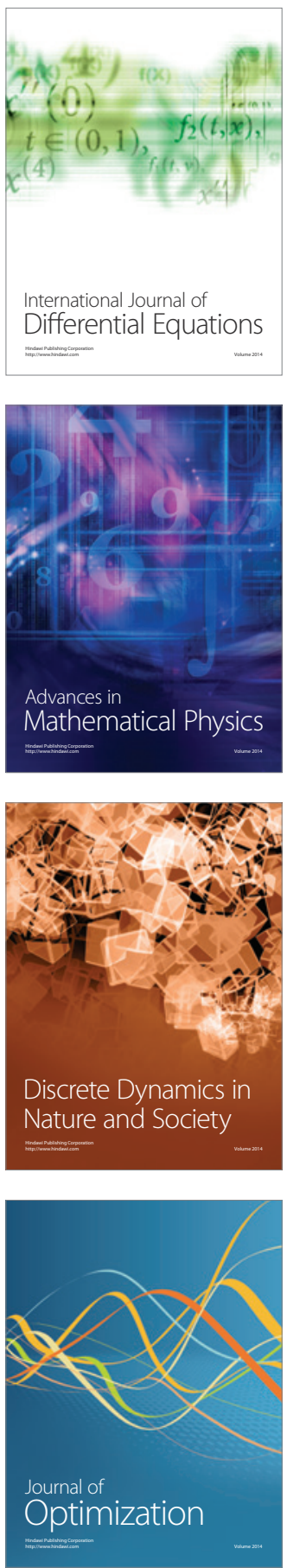